\title{
Sensitivity to diazepam after a single session of forced swim stress in weaning Wistar rats
}

\author{
BLANDINA BERNAL-MORALES ${ }^{1, *}$ \\ GABRIEL GUILLÉN-RUIZ ${ }^{1}$ \\ JONATHAN CUETO-ESCOBEDO ${ }^{1}$ \\ JUAN FRANCISCO RODRÍGUEZ-LANDA ${ }^{1}$ \\ CARLOS M. CONTRERAS ${ }^{1,2}$ \\ ${ }^{1}$ Laboratorio de Neurofarmacología \\ Instituto de Neuroetología \\ Universidad Veracruzana, Xalapa \\ México \\ ${ }^{2}$ Unidad Periférica Xalapa \\ Instituto de Investigaciones Biomédicas \\ Universidad Nacional Autónoma \\ de México, Instituto de Neuroetología \\ Universidad Veracruzana, Xalapa \\ México
}

Accepted March 18, 2018 Published online April 11, 2018

\begin{abstract}
The present study investigated the sensitivity to stress and diazepam in weaning (21-day old) Wistar rats. A single 15min session of forced swimming was used to induce anxiety-like behavior. The group that was forced to swim exhibited an increase in anxiety-like behavior in the elevated plus maze (EPM) and open field test (OFT) compared to the non-stressed group. Diazepam (1 $\mathrm{h}$ before the tests) reduced anxiety-like behavior in rats forced to swim compared to the vehicle stressed group. The dose-response curve for diazepam indicated that the $0.5 \mathrm{mg} \mathrm{kg}^{-1}$ dose $(1 \mathrm{~h}$ before the EPM and OFT) was the minimum effective dose in reducing anxiety-like behavior without altering locomotor activity in weaning rats. These results indicate that weaning rats can develop anxiety-like behavior after a brief, single session of stress, and that rats at this age are seemingly more sensitive to diazepam than adult rats, which may be taken into account for clinical applications.
\end{abstract}

Keywords: anxiety, diazepam, weaning rats, elevated plus maze, forced swim, open field test

Anxiety and depression are emotional disorders that are scarcely investigated in very young animals. The elevated plus maze (EPM) has been extensively used to explore behavioral responses to anxiolytic drugs (1). On postnatal days (PND) 34-39, rats spend more time on the open arms of the EPM than older rats (2), suggesting age-related differences in the expression of anxiety-like behavior. In the open field test (OFT), infant rats exhibit locomotor activity that is similar to adult rats when they are in familiar cages, but age-related differences in locomotor activity are observed when rats are exposed to a novel open field environment (3).

Diazepam is one of the most commonly used anxiolytic drugs. It is used as a reference anxiolytic drug at a minimal dose of $1-2 \mathrm{mg} \mathrm{kg}^{-1}$ in adult rats subjected to experimental models of anxiety (4). However, responses to stressful situations and subsequent responses to anxiolytic drugs have been scarcely explored in very young animals. Therefore, the aim

\footnotetext{
*Correspondence; e-mail: bbernal@uv.mx
} 
of the present study was to investigate the effects of a single 15-min session of forced swim stress on anxiety-like behavior in weaning rats on PND21 and to explore the possible differential potency of diazepam.

\section{EXPERIMENTAL}

\section{Animals}

The study included offspring of Wistar rats provided by the Biomedical Research Institute, National Autonomous University of Mexico (UNAM), Xalapa, Mexico. During gestation and after delivery, each dam and its litter (approximately 10 pups) remained in acrylic boxes $(44 \mathrm{~cm}$ length $\times 33 \mathrm{~cm}$ width $\times 20 \mathrm{~cm}$ height) under a $12 \mathrm{~h} / 12 \mathrm{~h}$ light/dark cycle (lights on at 7:00 a.m.) with free access to purified water and food (Teklad Lab Animal Diets, USA). The nest remained undisturbed, with minimal handling when changing the bedding material (every $48 \mathrm{~h}$ ). The offspring ( $45 \pm 5 \mathrm{~g}$ each) were weaned on PND21. They were then randomly assigned to eight experimental groups and subjected to behavioral tests. Each group had an approximately 1:1 proportion of males to females, with only 2-3 rats from the same litter assigned to the same group.

All procedures followed the principles of animal care based on the Guide for the Care and Use of Laboratory Animals (5) and Mexican law requirements. The experimental protocol received authorization from the Biomedical Research Institute Ethical Committee, National Autonomous University of Mexico (UNAM).

\section{Behavioral tests}

Elevated plus maze. - The elevated plus maze (EPM) was constructed of wood and situated in a white light illuminated room (40 lux). The maze consisted of two opposite open and closed arms set in a plus configuration. The apparatus was elevated $50 \mathrm{~cm}$ above the floor. Open arms (50 cm length $\times 10 \mathrm{~cm}$ width) were painted white. Closed arms $(50 \mathrm{~cm}$ length $\times 10 \mathrm{~cm}$ width $\times 40 \mathrm{~cm}$ height) were painted black. Rats were individually placed in the center of the maze, facing an open arm. The animals were free to explore the EPM for $5 \mathrm{~min}$. The EPM is used to assess anxiety-like behavior, in which higher "anxiety" is associated with less exploration of the open arms. We recorded the number of entries into and time spent on the open arms, which are indicators of exploratory activity in a situation commonly avoided by rats, which naturally prefer closed and dark places. We also calculated the anxiety index according to Cohen et al. (6). The anxiety index is calculated as the proportion of entries into and time spent on the open arms relative to the duration of the test and the total number of entries. A higher anxiety index reflects higher "anxiety." The EPM was cleaned with a $5 \%$ ethanol solution after each animal was tested. Behavioral data of animals that fell from the maze were excluded from the analyses.

Open field test. - After the EPM test, each infant rat underwent the open field test (OFT) for $5 \mathrm{~min}$ to evaluate the effects of treatments on spontaneous locomotor activity. The automatic motor activity monitor (Acti-Track v2.7.10, PanLab Instruments, S.L., Spain) consisted of a Perspex box ( $45 \mathrm{~cm}$ length $\times 45 \mathrm{~cm}$ width) with $35 \mathrm{~cm}$ high walls that contained a total of 32 infrared beams, 16 on each perpendicular wall. Infrared sensors were connected to 
an interface (LE 8811, LSI Letica Scientific Instruments, Spain) and a computer to record rats' movements. The floor of the cage was virtually divided into five zones (four peripheral zones and one central zone). The test lasted $5 \mathrm{~min}$. We recorded the number of entries into each zone (crossings) and the number of crossings in the central zone. Rats usually prefer to stay close to walls and avoid crossing the center of any area. A larger number of crossings in the central area reflects lower "anxiety" (7). No other behaviors, such as rearing or grooming, were evaluated. After each experimental session, the locomotor activity box was carefully cleaned with $5 \%$ ethanol.

\section{Diazepam dose-response study}

To determine the minimum anxiolytic dose of diazepam in infant rats at PND21, five groups of rats were taken directly from their housing facilities and received different treatments. The first group was the negative control that received $0.9 \% \mathrm{NaCl}$ (control, $n=15$ ), while the other four groups were injected with different doses of diazepam (Valium 10, Hoffman-La Roche, Switzerland): $0.1 \mathrm{mg} \mathrm{kg}^{-1}$ (DZP-0.1, $\left.n=11\right), 0.5 \mathrm{mg} \mathrm{kg}^{-1}$ (DZP-0.5, $n=12$ ), $1 \mathrm{mg} \mathrm{kg}^{-1}(\mathrm{DZP}-1, n=12)$, and $5 \mathrm{mg} \mathrm{kg}^{-1}$ (DZP-5, $\left.n=9\right)$. All injections were subcutaneous with a volume of $0.3 \mathrm{~mL}$ per rat. One hour after the injection, the rats were individually subjected to the EPM and OFT. All the procedures were performed during the light period between 11:00 a.m. and 3:00 p.m.

\section{Effect of diazepam in rats subjected to forced swim stress}

Another set of PND21 rats was assigned to three different groups. The first group received only vehicle $(0.9 \% \mathrm{NaCl})$ and was then subjected to the EPM and OFT (control, $n=$ 12). The second group underwent a single session of forced swim and received vehicle before it was subjected to the EPM and OFT (FS, $n=12$ ). The third group received diazepam $23 \mathrm{~h}$ after being forced to swim and was then subjected to the EPM and OFT (FS+DZP, $n=7$ ). One hour after the injection, the three groups were subjected to the EPM and OFT. The $0.5 \mathrm{mg}$ $\mathrm{kg}^{-1}$ dose of diazepam was chosen based on the aforementioned dose-response pilot study.

\section{Stress session}

The rats were individually placed in an acrylic cylinder $(49 \times 20 \mathrm{~cm})$ filled with clean water $\left(25 \pm 1^{\circ} \mathrm{C}\right)$ to a depth of $25 \mathrm{~cm}$ for $15 \mathrm{~min}$. At the end of the forced swim session, the rats were placed in a dry and clean cage and then returned to their housing facilities. The behavioral tests were conducted $24 \mathrm{~h}$ after this single forced swim session in which no behaviors were measured or recorded.

\section{Statistical analysis}

The data were analyzed using the Kruskal-Wallis test because the data followed a non-parametric distribution. When differences were detected, we used Dunn's multiplecomparison post hoc test and $p$-values $\leq 0.05$ were considered statistically significant. All statistical analyses were performed using Sigma Stat 3.5 software (Systat Software, Chicago, IL, USA). The results are presented as the $H$ test statistic, degrees of freedom, and $p$-values. The data are expressed as mean \pm standard error of the mean (SEM). 


\section{RESULTS AND DISCUSSION}

\section{Diazepam dose-response study}

Elevated plus maze. - The main aim of the present study was to determine the minimal anxiolytic dose of diazepam in non-stressed weaning rats. In the EPM, an increase in the time spent on the open arms reflects a low level of "anxiety," and an increase in the time spent on the closed arms reflects a high level of "anxiety," given that rats prefer to stay in enclosed and dark places (1). DZP-0.5, DZP-1 and DZP-5 increased significantly the time spent on the open arms of the EPM $\left(H_{4}=17.956, p<0.001\right)$ in PND21 rats compared to the control group (Table I). This effect is considered an anxiolytic-like effect in preclinical research, given that diverse clinically effective anxiolytic drugs increase this variable in the EPM. The $0.1 \mathrm{mg} \mathrm{kg}^{-1}$ dose of diazepam increased the time spent on the open arms, but this effect did not reach statistical significance. The minimal effective dose of diazepam that significantly reduced indicators of anxiety was $0.5 \mathrm{mg} \mathrm{kg}^{-1}$. Here, a ceiling effect was observed, since higher doses ( 1 and $5 \mathrm{mg} \mathrm{kg}^{-1}$ ) did not produce additional effects. The anxiety index integrates all variables of the EPM (i.e., total time on the EPM, time spent on the open arms, number of entries into the open arms, and total number of entries into the open arms). The anxiety index is a robust measure of anxiolytic action. The anxiety index decreases when an anxiolytic action is detected (6). A low anxiety index was observed in the DZP-0.5, DZP-1, and DZP-5 groups compared to the control group $\left(H_{4}=17.717, p<0.001\right)$, with no significant differences between doses higher than the minimal effective dose. A novel finding of the present study was that $0.5 \mathrm{mg} \mathrm{kg}^{-1}$ exerted an anxiolytic effect that appeared to be effective in weaning rats. The effective dose of diazepam in reducing indicators of anxiety in adult rats, as reported by our research group and some others, is 1-2 $\mathrm{mg} \mathrm{kg}^{-1}(4,8,9)$, and in contrast, as stated by other authors, doses from $0.1-0.625 \mathrm{mg} \mathrm{kg}^{-1}$ were ineffective to reduce anxiety in adult mice and rats $(9,10)$.

Open field test. - Locomotor activity in the OFT is commonly assessed in studies of anxiety and the actions of anxiolytic drugs. These can produce sedative actions to discard possible influences on locomotion (e.g., restlessness or freezing). In the present study, the DZP-5 group had the smallest number of total crossings $\left(H_{4}=11.214, p=0.024\right)$ and the smallest number of crossings in the central zone $\left(H_{4}=10.067, p=0.039\right)$ compared to other groups (control, DZP-0.1, DZP-0.5, and DZP-1; Table I). This hypoactivity illustrates a seda-

Table I. Dose-response study of behavior in the elevated plus maze and open field test in PND21 rats

\begin{tabular}{lcccccc}
\hline & Control & \multicolumn{5}{c}{ Diazepam dose $\left(\mathrm{mg} \mathrm{kg}^{-1}\right)$} \\
\hline & 0 & 0.1 & 0.5 & 1 & 5 & $p$ \\
\hline Open arm time (s) & $44.6 \pm 5.8$ & $84.1 \pm 16.0$ & $112.6 \pm 19.6^{*}$ & $106.9 \pm 15.1^{*}$ & $157.6 \pm 28.5^{*}$ & $<0.001$ \\
Anxiety index & $0.71 \pm 0.02$ & $0.63 \pm 0.04$ & $0.57 \pm 0.06^{*}$ & $0.54 \pm 0.02^{*}$ & $0.43 \pm 0.07^{*}$ & $<0.01$ \\
Total crossings & $26.8 \pm 2.4$ & $33.6 \pm 6.1$ & $26.2 \pm 3.5$ & $29.3 \pm 3.9$ & $15.2 \pm 3.1^{*}$ & $<0.024$ \\
Central zone crossings & $4.9 \pm 0.5$ & $5.0 \pm 1.1$ & $4.4 \pm 1.1$ & $4.9 \pm 1.1$ & $1.4 \pm 0.6^{*}$ & $<0.039$ \\
\hline
\end{tabular}

* Statistically significant difference vs. control group. 
tive effect of $5 \mathrm{mg} \mathrm{kg}^{-1}$ diazepam, an effect consistent with a previous study that found that the $5 \mathrm{mg} \mathrm{kg}^{-1}$ dose increased sedation scores in PND28 rats (11). This finding in the DZP-5 group suggests that $5 \mathrm{mg} \mathrm{kg}^{-1}$ should not be administered in weaning rats because it is sedative rather than anxiolytic. In contrast, a single dose of $2 \mathrm{mg} \mathrm{kg}^{-1}$ produced anxiolytic actions in adult rats without influencing locomotion (8). Therefore, non-stressed weaning rats respond to a dose of diazepam that is $25 \%$ of the effective anxiolytic dose in adult rats, without producing sedative actions.

\section{Stressed rats}

Elevated plus maze. - A single session of forced swimming is able to produce marked changes in neuronal firing in limbic structures 1 day after the first session (12). Therefore, in the present study, weaning rats were forced to swim and were behaviorally tested on the following day in a model of anxiety. Diazepam was selected as the anxiolytic drug at the minimally effective anxiolytic dose determined in the first experiment (i.e., $0.5 \mathrm{mg}$ $\mathrm{kg}^{-1}$ ), which was administered before the behavioral tests. The FS group exhibited a significant decrease in the time spent on the open arms $\left(H_{2}=22.271, p<0.001\right)$ compared to the control group. This decrease was reversed in the FS+DZP group, thus demonstrating an anxiolytic action (Fig. 1a). No differences were observed between the FS+DZP and control groups. The anxiety index was significantly higher in the FS group compared to the control and FS+DZP groups $\left(H_{2}=20.150, p<0.001\right.$; Fig. 1b). Our contribution to the literature consists of the use of forced swimming as an inducer of anxiety in weaning rats. We found that a lower dose of diazepam than that used in adult rats is sufficient to reduce anxietylike behavior produced by the stressful situation of being forced to swim in weaning rats. Forced swimming is an inescapable situation. We found that young rats were clearly sensitive to this stressor, supporting other observations in which pre-weaning rats exhibited species-specific stress responses that were related to anxiety (i.e., freezing and fewer vocalizations) when they were subjected to maternal isolation (13).

Open field test. - The FS group exhibited fewer total crossings in the OFT compared to the control group $\left(H_{2}=17.260, p<0.001\right)$. Diazepam administration in the FS+DZP group reversed the anxiogenic effect of swim stress (Fig. 2a). Decreases in locomotion have been

a)

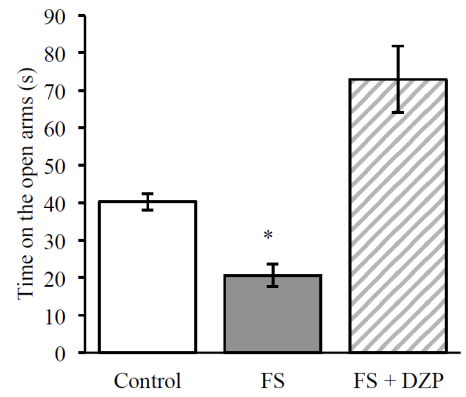

b)

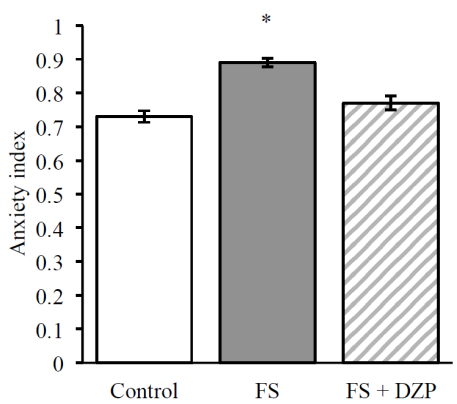

Fig. 1. Elevated plus maze results in rats forced to swim (FS) and injected with diazepam (DZP). Forced swimming: a) decreased the time spent on the open arms, b) increased the anxiety index, compared to the control group. Significant difference vs. control group: ${ }^{*} p \leq 0.05$. 

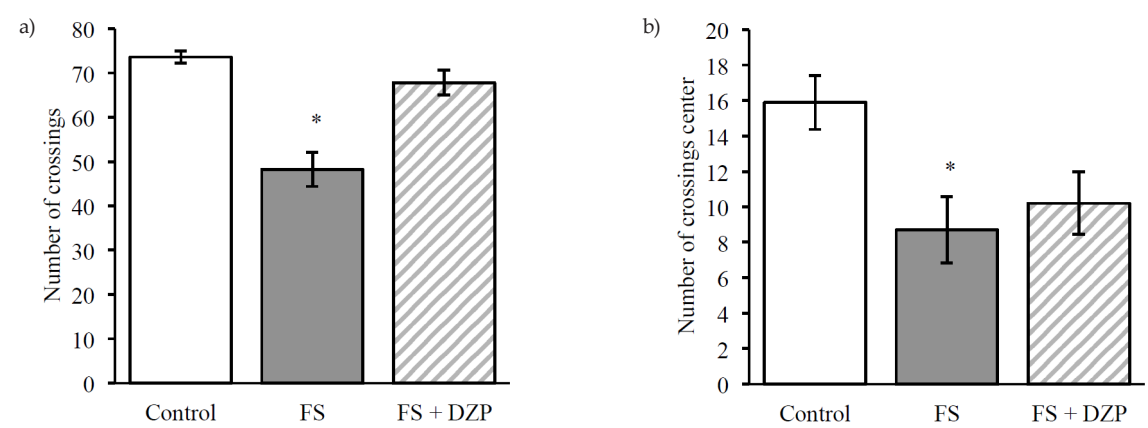

Fig. 2. Open field test results in rats forced to swim (FS) and injected with diazepam. Forced swimming: a) decreased the total number of crossings, b) decreased the number of crossings in the central zone, compared to the control group. Significant difference vs. control group: ${ }^{*} p \leq 0.05$.

observed when rats or mice are subjected to stressful situations, which is correlated with anxiety-like behavior in various experimental models of anxiety. Rats in the FS group that were forced to swim made $~ 55 \%$ fewer crossings in the central zone compared to the control group $\left(H_{2}=7.609, p<0.022\right)$, and this effect was reduced by diazepam (Fig. $\left.2 b\right)$. This observation is consistent with other studies that found a reduction of central crossings in the OFT in "anxious" animals (8). Diazepam increased locomotion in the central zone in the FS+DZP group, thus reflecting a lower state of anxiety (8). These results suggest that a single session of forced swimming produced clear anxiogenic-like effects in the OFT, an effect that was absent in non-stressed rats, which allowed assessment of anxiolytic actions of diazepam. Notably, the effects of drugs may be different in animals that come directly from their home cages compared to animals that are subjected to stress before testing.

Forced swimming is generally used to measure antidepressant actions and despair, but our results indicate that it is also useful for producing anxiety. Therefore, in agreement with other authors, forced swimming should be considered in studies of stress and related disorders beyond depression (14). In humans, anxiety-related disorders are strongly linked to stress exposure, which can affect adults, adolescents and children and be comorbid with depression (15). Studies of the therapeutic potential of pharmacological strategies to control anxiety have been mainly conducted in adults, thus failing to develop or evaluate such pharmacological strategies for the treatment of anxiety symptoms in young individuals.

\section{CONCLUSIONS}

A single session of forced swimming was an aversive stimulus that produced anxiogenic-like effects in weaning rats. This finding supports the concept that an early stressor impacts the emotional state of young rats. Young rats were sensitive to the actions of the anxiolytic drug diazepam at a dose that is typically ineffective in older rats. Administration of low-dose anxiolytics in very young subjects demands new schedules of drug administration to be developed. Such revised administration regimens may avoid potential side effects and development of pharmacological resistance to or dependence on benzodiazepines, but further studies are needed to investigate this possibility. 
This study evaluated the effect of a stressful situation on anxiety-like behavior in young PND21 rats. The present findings may impact the design of new experiments that evaluate pharmacological strategies to ameliorate anxiety symptoms in very young individuals. We found that only $25 \%$ of the effective dose of diazepam that is used in adult rats was sufficient to significantly reduce anxiety-like behavior in young rats. We suggest that young and adult individuals exhibit differential sensitivity to anxiolytic drugs. Preventing the use of excessive or unnecessary doses of anxiolytic drugs may avoid side effects in young patients.

Acknowledgments. - This study was supported by a grant from the Consejo Nacional de Ciencia y Tecnología (CONACyT: CB- 2006-1, 61741), Universidad Nacional Autónoma de México (DGAPAPAPIIT IN211111-3), and Universidad Veracruzana (UVE-CA-25).

Jonathan Cueto-Escobedo and Gabriel Guillén-Ruiz received fellowships from CONACyT (Reg. 197556 and 249708). The authors thank Michael Arends for revising and editing the English of this manuscript.

\section{REFERENCES}

1. M. Bourin, Animal models for screening anxiolytic-like drugs: a perspective, Dialogues Clin. Neurosci. 17 (2015) 295-303.

2. D. A. Lynn and G. R. Brown, The ontogeny of anxiety-like behavior in rats from adolescence to adulthood, Dev. Psychobiol. 52 (2010) 731-739; https://doi.org/10.1002/dev.20468

3. K. S. Smith and J. I. Morrell, Comparison of infant and adult rats in exploratory activity, diurnal patterns, and responses to novel and anxiety-provoking environments, Behav. Neurosci. 121 (2007) 449-461; https://doi.org/10.1037/0735-7044.121.3.449

4. F. Mohammad, J. Ho, J. H. Woo, C. L. Lim, D. J. J. Poon, B. Lamba and A. Claridge-Chang, Concordance and incongruence in preclinical anxiety models: systematic review and meta-analyses, Neurosci. Biobehav. Rev. 68 (2016) 504-529; https://doi.org/10.1016/j.neubiorev.2016.04.011

5. National Research Council, Guide for the Care and Use of Laboratory Animals, 7th ed., National Academy Press, Washington (DC) 1996.

6. H. Cohen, M. A. Matar and Z. Joseph, Animal models of post-traumatic stress disorder, Curr. Protoc. Neurosci. Chapter 9 (2013) Unit 9.45; https://doi.org/10.1002/0471142301.ns0945s64

7. L. Prut and C. Belzung, The open field as a paradigm to measure the effects of drugs on anxietylike behaviors: a review, Eur. J. Pharmacol. 463 (2003) 3-33; https://doi.org/10.1016/S00142999(03)01272-X

8. C. M. Contreras, J. F. Rodríguez-Landa, R. I. García-Ríos, J. Cueto-Escobedo, B. Guillén-Ruiz and B. Bernal-Morales, Myristic acid produces anxiolytic-like effects in Wistar rats in the elevated plus maze, BioMed. Res. Int. 2014 (2014) Article ID 492141 (8 pages); https://doi.org/10.1155/2014/492141

9. R. Ravenelle, A. K. Berman, J. La, B. Mason, E. Asumadu, C. Yelleswarapu and S. T. Donaldson, Sex matters: females in proestrus show greater diazepam anxiolysis and brain-derived neurotrophic factor- and parvalbumin-positive neurons than males, Eur. J. Neurosci. (2018) in press; https://doi.org/10.1111/ejn.13870

10. J. Simpson, C. Ryan, A. Curley, J. Mulcaire and J. P. Kelly, Sex differences in baseline and druginduced behavioural responses in classical behavioural tests, Prog. Neuropsychopharmacol. Biol. Psychiatry 37 (2012) 227-236; https://doi.org/10.1016/j.pnpbp.2012.02.004A

11. G. Guillén-Ruiz, B. Bernal-Morales, C. M. Contreras, J. Cueto-Escobedo and J. F. Rodríguez-Landa, Oleic acid produces motor incoordination and hypoactivity in infant Wistar rats through $\mathrm{GABA}_{\mathrm{A}}$ receptors, Am. J. Psychiatry Neurosci. 4 (2016) 18-25; https://doi.org/10.11648/j.ajpn.20160402.11 
12. C. M. Contreras, L. Chacón, J. F. Rodríguez-Landa, B. Bernal-Morales, A. G. Gutiérrez-García and M. Saavedra, Spontaneous firing rate of lateral septal neurons decreases after forced swimming test in Wistar rat, Prog. Neurospychopharmacol. Biol. Psychiatry 28 (2004) 343-348; https://doi. org/10.1016/j.pnpbp.2003.10.013

13. S. A. Brunelli, J. P. Curley, K. Gudsnuk, F. A. Champagne, M. M. Myers, M. A. Hofer and M. G. Welch, Variations in maternal behavior in rats selected for infant ultrasonic vocalization in isolation, Horm. Behav. 75 (2015) 78-83; https://doi.org/10.1016/j.yhbeh.2015.08.007

14. K. G. Commons, A. B. Cholanians, J. A. Babb and D. G. Ehlinger, The rodent forced swim test measures stress-coping strategy, not depression-like behavior, ACS Chem. Neurosci. 8 (2017) 955960; https://doi: 10.1021/acschemneuro.7b00042

15. M. G. Craske and M. B. Stein, Anxiety, Lancet 388 (2016) 3048-3059; https://doi.org/10.1016/S01406736(16)30381-6 